\title{
Acute Upper Limb Ischemia after an Accidental Intra-Arterial Antispasmodic Injection on an Anatomical Variant of the Radial Artery: A Case Report and Review of the Literature
}

\author{
Elfatemi B*, Bakkali T, Lazraq M, Khloufi S, Idrissi R, Sefiani Y, Lekehel B, Mesnaoui A, Ammar F, Bensaid Y
}

Vascular Surgery, Cardiology Department of the Ibn Sina Rabat Hospital

DOI: $10.36347 /$ simcr.2020.v08i03.011

| Received: 29.02.2020 | Accepted: 07.03.2020 | Published: 10.03.2020

*Corresponding author: Elfatemi Bouthainah

Abstract

Accidental intra-arterial injection of various drugs, particularly antibiotics and phenobartbiturates and the occurrence of acute distal ischemia have been reported in the literature. However, no publication has explained the embologenic mechanism of antispasmodics. Most cases reported involved children and the injection site was the brachial artery. We report the observation of a patient having had distal ischemia of the hand after injection of antispasmodic in the posterior branch of the radial artery, the division of which is subject to anatomical variation.

Keywords: Radial artery-anatomical variant-intra-arterial injection-acute upper limb ischemia-antispasmodic.

Copyright @ 2020: This is an open-access article distributed under the terms of the Creative Commons Attribution license which permits unrestricted use, distribution, and reproduction in any medium for non-commercial use (NonCommercial, or CC-BY-NC) provided the original author and source are credited.

\section{INTRODUCTION}

Radial artery takes route from the back of the forearm and ends at the level of the wrist in two branches, one behind the arcade of the palm whilst the other winds around the exterior frontier of the wrist, becoming frontal, moving in the anatomical snuff box in order to join the first intercostal space where it ends. Extreme distal ischemia resulting from accidental intravenous injection with diverse medicines has been the object of several publications. Among these substances, one may mention antibiotics and phenobarbiturics but no antispasmodic case has been reported. We report the observation of a patient who contracted an acute ischemia after 6 hours of accidental antispasmodic injection at the level of the posterior branch of the radial artery, which in the case of our patient splits at the level of the lower third of the forearm (anatomical variant), thereby confusing it with the cephalic vein of the forearm.

\section{OBSERVATION}

A 70-year-old patient regularly follow-up for renal lithiasis was admitted urgently for an attack of renal colic. A prescription of venous antispasmodic medication was given to the nurse on duty, who found a good calibre vessel traveling on the posterior surface of the lower third of the arm, judged that it was the cephalic vein in the forearm, and injected a dose of antispasmodic medication to relieve the patient's pain, a small bandage was applied after removal of the tourniquet and the patient came back home. 6 hours later the patient felt acute pain in the hand with pale coldness and cyanosis of the extremities requiring urgent vascular advice. Vascular examination finds brachial and ulnar pulses present with absence of the radial pulse, a cyanotic appearance of the lower third of the forearm and finger pulps (Figure 1). An emergency Doppler ultrasound exam was carried out to visualize the leak of vascular flow in the lower third of the radial artery. The vascular medical team decided to treat the patient with heparin at the effective dose. 24 hours later, the repermeabilization of the radial artery was noticed, as the pulse came back, and a pulse on the dorsal surface of the forearm on the path of the cephalic vein was noticed too. A Doppler exam was carried out to see the upper birth of the posterior branch of the radial artery in the lower third of the forearm instead of the outer end of the wrist as described in anatomy books. This branch continues after a normal journey to the anatomical snuffbox (Figure 2).

The patient underwent a curative anticoagulant therapy for two weeks. 15 days later, the clinical examination revealed that the necrosis was limited to the distal end of the index finger and thumb, which was regularized with good healing. 




Fig-1: Acute ischemia of the upper limb with distal necrosis after antispasmodic injection in intra arterial

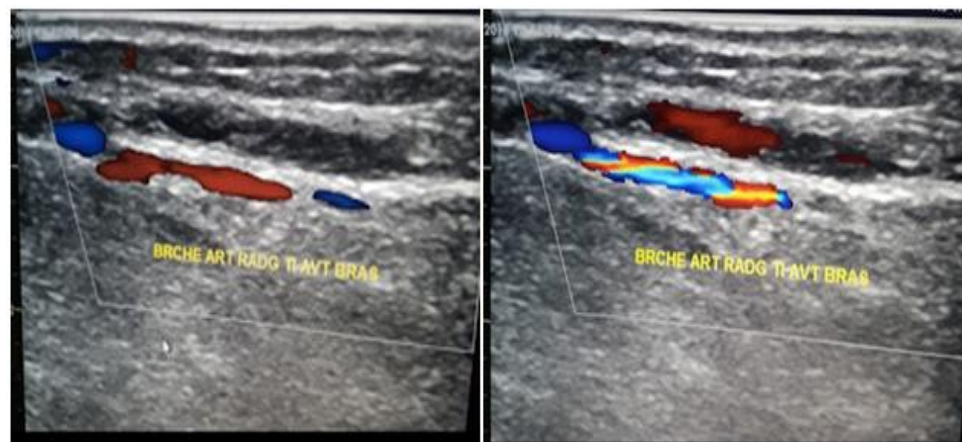

Fig-2: Ultrasonographic appearance of the posterior branch of the radial artery which divides higher than the external surface of the wrist

\section{DisCUSSION}

The radial artery is a branch of the external bifurcation of the humeral artery; it goes from the fold of the elbow to the palm of the hand. Usually less voluminous than the ulnar, the radial first goes obliquely downwards and outwards, almost following the same direction of the humerus; then it goes vertically to above the radiocarpal space, where it deflects outwards and backwards and, below the styloid process of the radius, and bypasses the external lateral ligament of the radiocarpal joint. Then, after having circulated from the anterior face of the forearm to the external face of the wrist, it obliquely reaches the first intermetacarpal space, in the upper end of which it sinks to reach the palm, where it will form, by anastomosing with a branch of the ulnar bone, the deep palmar arch.

There are different anatomical variants: variations in its origin, its collateral branches regarding the trunk of the interosseous and at the level of its bifurcation regarding the palmar arch, the early high division of its posterior branch is not described in the literature, and was observed in this patient.

Accidental intra-arterial injection is extremely rare. Only a very few cases have been published in the literature reporting the occurrence of distal acute ischemia after an intra-arterial antibiotic injection, the first observation was reported in 1960 by Knowels [1].
Several pathophysiological hypotheses have been put forward concerning penicillin: the product seems to be implicated in the formation of microcrystals in small vessels, intimal lesions could lead to hemolysis associated with platelet aggregation, venous obstruction would lead to stasis and thrombosis, and finally vasospasm could be reflex [2]. Clinically, acute pain remains the main symptom, followed by signs of ischemia, and must enable the physician to set the diagnosis.

There is no universally accepted treatment protocol. Some authors recommend limb elevation and massage in combination with heparin therapy [3]. Others suggest the use of lidocaine $[4,5]$ or hyperbaric oxygen therapy [6]. In some cases, regional nerve blocks or sympathectomy have been performed without much more success [7, 3]. Good results have been obtained with intra-arterial injection of iloprost [4, 8], and in one observation Bonnie used papaverine intraarterially successfully [3]. In this case the anatomical variant of the radial artery, which is the upper division of the radial artery in the lower third of the forearm, was the cause of the mistake during the injection, because this arterial branch was confused with the cephalic vein of the forearm, one could not perceive the pulse because of the tourniquet. The mechanism of action of antispasmodics in the occurrence of acute ischemia has not been yet determined as has been advanced for antibiotics and phenol barbiturates. 


\section{Conclusion}

The high division of the radial artery is extremely rare and could be in some cases a cause of paramedical error concerning the administration of parenteral drugs, accidentally intra-arterial, involving the functional prognosis of patients admitted for the follow-up of benign pathologies.

\section{REFERENCE}

1. Knowles JA. Accidental intra-arterial injection of penicillin. American Journal of Diseases of Children. 1966, $111: 552-6$

2. Aghoutane EM. Gangrène des doigts après injection accidentelle en intra-artériel de floxacilline: à propos d'un cas. Chirurgie de la main.2011; 30: 120-122

3. Bhabra MS, Meshikhes AN, Thomson GJL. Intraarterial temazepam: an importantcause of limb ischemia in intravenous drug abusers. Eur $\mathbf{J}$ Vasc Surg. 1994; 8:240-2.
4. Keene JR, Buckley KM, Small S. Accidental intraarterial injection: a case report,new treatment modalities, and a review of literature. J Oral Maxillofac Surg. 2006; 64:965-8.

5. Ali MS, Chowdhary UM, Mohanna. Drug induced arterial spasm releaved by lidocaine. Acta Chir Scand. $1986 ; 152: 697-9$.

6. Adir Y, Halpern P, Nachum Z. Hyperbaric oxygen therapy for ischemia of the hand due to intraarterial injection of methadone and flunitrazepam. Eur J Vasc Surg. 1991;5: 6779.

7. Arquilla B, Gupta R, Gernshiemer J. Acute arterial spasm in an extremity caused by inadvertent intraarterial injection successfully treated in the emergency department. J Emerg Med. 2000; 19:139-43.

8. Jean-Franc, ois H. Geschwind MD, Mandeep S. Dagli, MD, Drew L. Lambert, MD and Hicham Kobeiter, MD; Department of Radiology, The Johns Hopkins Hospital, Baltimore,Maryland, USA, J Endovasc Ther. $2003 ; 10: 590-594$. 